\title{
Isolation and Identification of a New Clostridium Butyricum XYB11Strain Producing 1, 3-Propandiol from Soil
}

\author{
Yan-bo XUE ${ }^{1}$, Miao-miao $\mathrm{YANG}^{1}$, Jun-hua $\mathrm{YUN}^{1}$, Tinashe \\ Archbold MAGOCHA ${ }^{1}$, Yi-lin $\mathrm{QI}^{2}$, Lin-sen $\mathrm{YANG}^{3}$, Ming-ming GUO', \\ Fan Zou ${ }^{1}$ and Xiang-hui $\mathrm{Q}^{1,{ }^{*}}$ \\ ${ }^{1}$ School of Food and Biological Engineering, Jiangsu University, Zhenjiang 212013, \\ China \\ ${ }^{2}$ College of Science and Technology, Agricultural University of Hebei, Cangzhou \\ 061100, China \\ ${ }^{3}$ Key Lab of Conservation Biology for Shennongjia Golden Monkey,Administration \\ of Shennongjia National Park, Hubei 442421,China \\ ${ }^{*}$ Corresponding author
}

Keywords: Isolation, Identification, Clostridium butyricum, 1,3-propanediol.

\begin{abstract}
PD) is an important chemical material, which is wildly used in many fields. Clostridium butyricumis key strain which can transform glycerol into 1,3-PD. In this study, a new strain producing 1,3-PD was isolated from soil. On the basis of morphology, biochemical-physiological characteristics, this strain was preliminarily identified as $C$. butyricum. Then, the molecular method was used in the further identification. The $16 \mathrm{~S}$ rDNA sequence analysis showed that this strain performed $99 \%$ homology with $C$. butyricum. So this strain was identified as $C$. butyricum finally, and named as $C$. butyricum XYB11. This strain will give more choice for the strains usage in the biosynthesis of 1,3-PD.
\end{abstract}

\section{Introduction}

1, 3-propanediol (1, 3-PD) is a widely-used organic synthetic compound, which can be utilized as antifreeze, detergent, preservative, emulsifier, flavoring agent in industry and food fields. Because of the more extensively application, 1,3-PD is considered as the most potential chemical in the 21 th century. So much focus has been attracted into the study of 1, 3-PD production.

So far, there are many studies on the microbial fermentation of 1,3-PD. The metabolic pathway, by which glycerol is transferred to 1,3-PD, exists in many microorganisms such as Klebsiella pheumoniae, Clostridium butyricum, Lactobacillus reuteri and Citrobacter freundii. Comparing with, other strains, C. butyricum obviously possess more potential for high substrate tolerance, less by-products and other advantages[1]. In addition, in most microorganisms, glycerol is metabolized via a reductive branch which leads to 1,3-PD formation under anaerobic conditions. In this branch, glycerol is converted to 3-hydroxypropionaldehyde (3-HPA)by glycerol dehydratase (GDHt). Afterwards, 3-HPA is reduced to 1,3-PD by NADH-dependent 1,3-PD dehydrogenase. GDHt can dominate the decomposition of glycerol, whose role is very vital in this reductive branch. GDHt is coenzyme B12-dependent in most bacteria, while in $C$. butyricum, GDHt is $\mathrm{B}_{12}$-independent. So, the costly coenzyme $\mathrm{B}_{12}$ is avoided, which reduces the production cost of 1,3-PD vastly. More important, $C$. butyricumas one of the most important probioticsis very helpful for human beings. 
According to the study of Yang Jinmei[2], the growth of cancer cell would be repressed by the composition of $C$. butyricum or metabolites produced in a certain environment. It was reported that $C$. butyricumhas already been used to cure some indigestion sufferers, and it was made into a kind of microecological modulator in Japan last century. With all the biological function and intricate characteristics of microecology, $C$. butyricum has gain more attention by the researchers from many fields. Due to above advantages, $C$. butyricum is a good candidate strain for the biosynthesis of 1, 3-PD [3].

In this study, a stain of $C$. butyricum was isolated from soil sample, and was identified by morphological, physiological and biochemical characteristics and $16 \mathrm{~S}$ rDNA sequencing.

\section{Materials and Methods}

\section{Media and Materials}

Reinforced Clostridium Medium (RCM) contained the following components per liter of distilled water: Yeast extract powder3g, Beef extract $10 \mathrm{~g}$, Tryptone 10g, Glucose $10 \mathrm{~g}$, starch $1 \mathrm{~g}, \mathrm{NaCl} 5 \mathrm{~g}$, sodium acetate $3 \mathrm{~g}$, cysteine hydrochloride $0.15 \mathrm{~g}$, ager $15 \mathrm{~g}$ (if solid medium is necessary). TSN medium contained the following components per liter of distilled water: Tryptone $10 \mathrm{~g}$, Yeast extract $3 \mathrm{~g}$, sodium sulfite $10 \mathrm{~g}$, iron(III) citrate tribasic hydrate $0.5 \mathrm{~g}$, Novobiocin sodium salt $0.02 \mathrm{~g}$, polymyxin B Sulfate $0.05 \mathrm{~g}$. Medium of gelatin liquefaction contained the following components per liter of distilled water: peptone $5 \mathrm{~g}$, gelatin $100-150 \mathrm{~g}$. (The initial $\mathrm{pH}$ at 7.2-7.4 regulated by $\mathrm{NaOH}$ ). Medium for fermentation of carbohydrate contained the following components per liter of distilled water: Trypticase $10 \mathrm{~g}$, Carbohydrate $5 \mathrm{~g}$, Sodium Chloride 5g, Phenol red $0.189 \mathrm{mg}$. Fermentation of 1,3-PD was used $200 \mathrm{ml}$ RCM medium adding with $8 \mathrm{~g}$ glycerol in $500 \mathrm{ml}$ flask for culture $24 \mathrm{~h}$ at $37{ }^{\circ} \mathrm{C}$. Soil samples was collected from the campus of Jiangsu University, China.

\section{Enrichment and Isolation}

$90 \mathrm{ml}$ of sterilized distilled water containing $10 \mathrm{~g}$ soil sample was stirred until homogenous, then heated in a water bath at $80^{\circ} \mathrm{C}$ for $30 \mathrm{~min}$. The mixture was put to $50 \mathrm{ml} \mathrm{RCM}$ medium and incubated under anaerobic condition for $24 \mathrm{~h}$. Each culture was transfer into TSN medium for another $24 \mathrm{~h}$. Then the culture was gradient diluted and spread to the RCM medium for extra $24 \mathrm{~h}$. Typical milky white colonies with similar characteristics described in Bergey's Manual of Determinative Bacteriology were re-streaked on RCM for aerobic and anaerobic parallel culture for $48 \mathrm{~h}$. The cultures which had grown in anaerobic condition were used for following procedures and preservation [4].

\section{Morphology Physiology and Biochemistry}

Microscopic morphology was observed using light microscope. Gram staining, gelatin hydrolysis test and carbohydrate fermentation test were conducted according to Bergey's Manual of Determinative Bacteriology.

\section{Molecular Biology Identification}

The colony PCR was carried out in a mixture containing: $12.5 \mu \mathrm{L} 2 \times$ taq master 
$\operatorname{mix}($ dye plus), $1 \mu \mathrm{L}$ each of forward and reverse primers (final concentration 0.1 $\mathrm{mM}), 2 \mu \mathrm{L}$ template strain culture and deionized water to a final volume of $25 \mu \mathrm{L}$. This mixture containing all of the above was then transferred to a thermocycler (T100 Thermal Cycler, BIO-RAD). The universal primer pair contains: upstream primer (named 27F), 5'- AGAGTTTGATCCTGGCTCAG -3'; downstream primer (named 1492R), 5'- TACGGCTACCTTGTTACGACTT -3'. The reaction cycle for PCR consisted of an initial denaturation at $95{ }^{\circ} \mathrm{C}$ for $5 \mathrm{~min}, 34$ cycles of denaturation at 94 ${ }^{\circ} \mathrm{C}$ for $30 \mathrm{~s}$, annealing at $60{ }^{\circ} \mathrm{C}$ for $30 \mathrm{~s}$, and extension at $72{ }^{\circ} \mathrm{C}$ for $60 \mathrm{~s}$. The final extension was conducted at $72{ }^{\circ} \mathrm{C}$ for $10 \mathrm{~min}$ and then infinitely hold at $12{ }^{\circ} \mathrm{C}$ [5].The amplified DNA fragment waschecked by electrophoresis in $1 \%$ agarose gel. DL 5000 DNA marker was used in the electrophoresis. The PCR product was sequenced by Synbio Biotechnologies Co. Ltd. (China). Then BLAST was used in the identificationbase on the $16 \mathrm{~S}$ rDNA sequencing. Using the neighbor-joining method through MEGA 5.0 software, phylogenetic tree was built and bootstrap analysis was performed for 500 replicates to estimate the reliability of the tree topologies.

\section{Capability of Producing 1, 3-PD}

Products 1, 3-PD was detected by high-performance liquid chromatography (HPLC) system with an Ultimate XB-NH $\mathrm{NH}_{2}$ HPLC Column (Welch, China).

\section{Results and Discussions}

\section{Morphology physiology and biochemistry characteristics}

In the preliminary screening, water bathing was to remove the microoganismwithout spore, and antibiotic was used to wipe out the Gram-negative strains. After preliminary screening, 13 strains was obtained by morphology characteristics and 10 stains among them were excluded by the gram staining and gelatin hydrolysis. Thereafter, 1 strain named XYB11 was completely matched the characteristics of the Bergey's Manual of Determinative Bacteriology through the test of carbohydrate fermentation (Table 1). Furthermore, after 36 hours of culture, XYB11 strain could grow and forminto 1-3 mm diameter milky white colony with a small serrated edge (Fig. 1). In addition, this grampositive strain is rod-shaped under the microscope and capable of producing 1,3-PD. All the above characteristics of this strain were consistent with those of $C$. butyricum. So it was identified as $C$. butyricum preliminarily. 


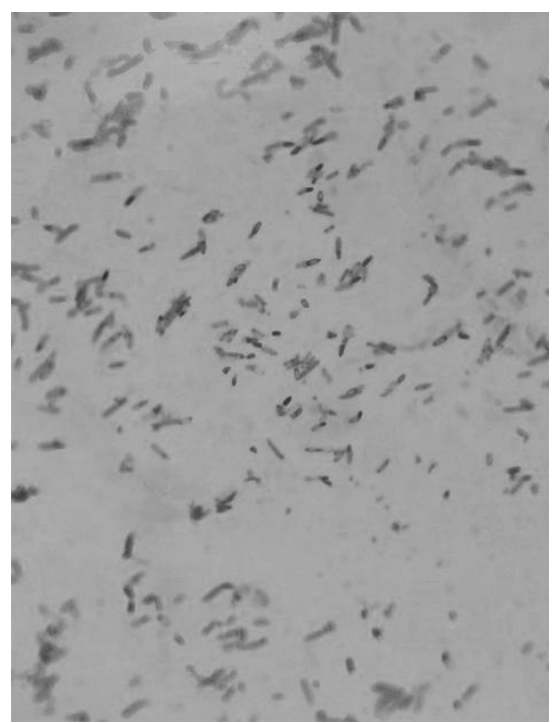

Figure.1 Colony morphology of strain XYB11

Table 1. Physiology and biochemistry characteristics of XYB11

\begin{tabular}{|c|c|}
\hline Test & result \\
\hline Gram staining & positive \\
\hline Gelatin hydrolysis & negative \\
\hline Starch & positive \\
\hline Glycerol & positive \\
\hline Melibiose & positive \\
\hline Melezitose & negative \\
\hline
\end{tabular}

\section{Molecular Biology Characteristics}

In order to further identify XYB11strain, the 16s rDNA sequence was determined through molecular method. A fragment about $1.5 \mathrm{Kbwas}$ obtained through colony PCR (Fig. 2). The BLASTresult showed that the 16s rDNA of XYB11strainshared 99\% identity to that of $C$. butyricum $\mathrm{BOH} 3$. Thephylogenetic tree onstructed by neighbor-joining method also showed that this strain is $C$. butyricum(Fig.3). So this stain was named as $C$. butyricumXYB11 finally.

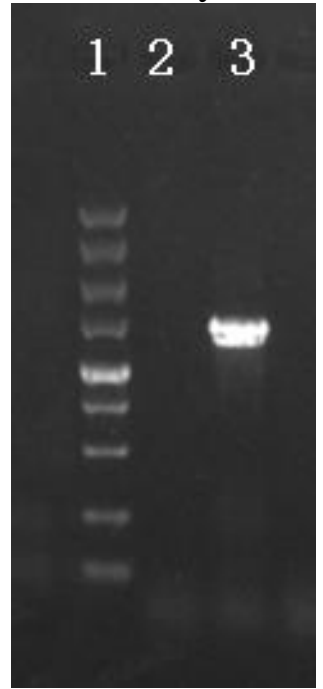

Figure.2PCR product of $16 \mathrm{~S}$ rDNA 


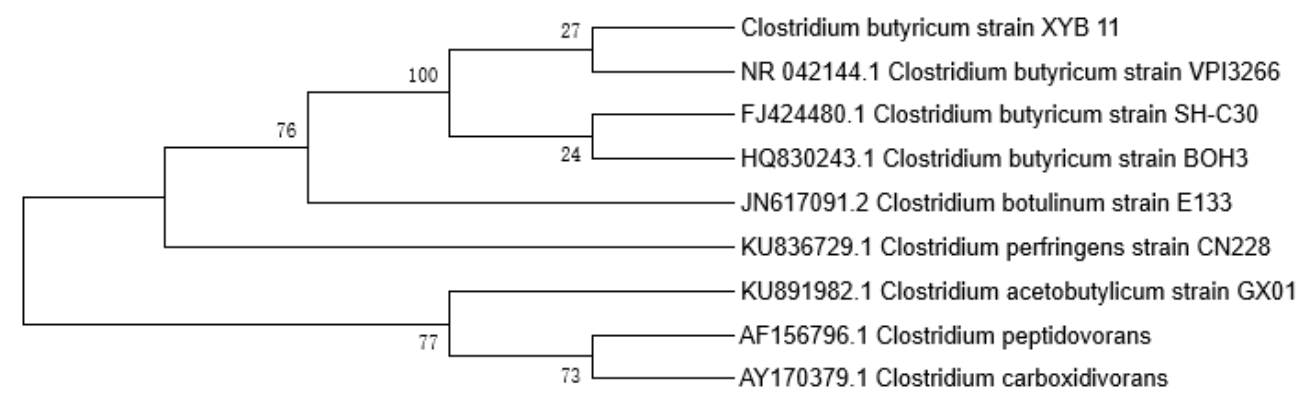

Figure. 3 The Phylogenetic tree analysis of XYB11

\section{Capability of Producing 1,3-PD}

RCM medium with $30 \mathrm{~g}$ glycerol was used for the 1,3-PD fermentation. After $24 \mathrm{~h}$ fermentation, $1.3 \mathrm{~g}$ of 1,3-PD was produced by C. butyricumXYB11, and the yield of $1,3-P D$ from glycerol was $0.16 \mathrm{~g} / \mathrm{g}$. The isolated strain of $C$. butyricumXYB11 had the capacity of producing 1,3-PD.

\section{Conclusion}

Via preliminary screening based on morphology, physiology and biochemistry, a gram-negative strain named XYB11 with rod-shape was obtained from the soil sample. Subsequently, this strain was further identified as $C$. butyricum XYB11 by 16S rDNA sequencing and BLAST.This strain had the capability of producing 1 , 3-PD.

\section{Acknowledgment}

This work was supported by the National Natural Science Foundation of China (No. 31571806) and and Scientific Research Promotion Fund of Jiangsu Province (Nos. 201610299012Z and 08JDG009) and PAPD.

\section{References}

[1] Z. Xiu, L. Jiang, H. Fu, et al., New progress in microbial fermentation of 1, 3-propanediol, J. Microbiol., 36 (2016) 1-9.

[2] J. Yang, Isolation and identification of Clostridium butyricum and the function and mechanism of colon cancer research, Chongqing Medical University, China, 2014.

[3]S. Saran, Microbial production of 1,3-propanediol: Recent developments and emerging opportunities, Biotechnol. Advances. 27 (2009):895-913.

[4] J. Zhao H.Zhang, F. Tian. Isolation, identification and selection of Clostridium butyricum [J]. Journal of Wuxi University of Light Industry, 21(2002)597-601.

[5] J. Wojtacka, B. Wysok, Z. Lipiński, et al., Clostridium botulinumspores found in honey from small apiaries in Poland, J. Agri. Sci. 60(2016) 89-100. 